\title{
Fecal carriage of CTX-M $\beta$-lactamase-producing Enterobacteriaceae in nursing homes in the Kinki region of Japan
}

This article was published in the following Dove Press journal: Infection and Drug Resistance 19 July 2013

Number of times this article has been viewed

\author{
Ulzii-Orshikh Luvsansharav' \\ Itaru Hirai' \\ Marie Niki' \\ Arisa Nakata' \\ Aya Yoshinaga' \\ Akira Yamamoto ${ }^{2}$ \\ Mayumi Yamamoto ${ }^{3}$ \\ Hiroyuki Toyoshima ${ }^{4, \dagger}$ \\ Fusao Kawakami ${ }^{5}$ \\ Nariaki Matsuura ${ }^{6}$ \\ Yoshimasa Yamamoto ${ }^{1,7,8}$ \\ 'Department of Bioinformatics, \\ Osaka University Graduate School \\ of Medicine, Osaka, ${ }^{2}$ Blueberry, \\ Amagasaki Health Care Facilities \\ for the Elderly, Hyogo, ${ }^{3}$ Health \\ Administration Center, Gifu \\ University, Gifu, ${ }^{4}$ Nursing home \\ Minoh, Osaka, ${ }^{5}$ Nursing home Itami, \\ Hyogo, ${ }^{6}$ Department of Molecular \\ Pathology, Osaka University Graduate \\ School of Medicine, Osaka, ${ }^{7}$ Osaka \\ Prefectural Institute of Public Health, \\ Osaka, ${ }^{8}$ Osaka University Global \\ Collaboration Center, Osaka, Japan
}

${ }^{\dagger}$ Hiroyuki Toyoshima passed away on February 21, 2013

Correspondence: Yoshimasa Yamamoto Osaka Prefectural Institute of Public Health, Nakamichi I-3-69, Higashinari-ku, Osaka, 537-0025, Japan

$\mathrm{Tel}+8$ I 66972 । 32 I

Fax +8I 669722393

Email yyamamot@iph.pref.osaka.jp
Abstract: The detection rate of CTX-M-type $\beta$-lactamase-producing Enterobacteriaceae in Japan has significantly increased. Nursing homes may be a reservoir of antibiotic-resistant bacteria. Therefore, we determined the prevalence of, and risk factors associated with, fecal carriage of CTX-M-type $\beta$-lactamase-producing Enterobacteriaceae among nursing home residents. A total of 225 stool samples were collected for phenotypic and genotypic identification of extended-spectrum $\beta$-lactamase (ESBL)-producing Enterobacteriaceae. Multivariate analysis was performed to identify the risk factors associated with fecal carriage of CTX-M producers. The prevalence of CTX-M-type ESBL-producing Enterobacteriaceae, as confirmed by phenotypic and genotypic methods, was 19.6\% (44 of 225 samples). Escherichia coli was the predominant CTX-M-type ESBL-producing bacterium among these isolates (41 of 44 isolates). Genotyping of bla $a_{\text {СТХ-м }}$ gene-positive isolates showed that $30(68.2 \%)$, $13(29.5 \%)$, and 1 (2.3\%) of 44 samples belonged to groups CTX-M-9, CTX-M-1 and CTXM-2, respectively. Among the CTX-M-type ESBL-producing Enterobacteriaceae found in nursing homes, $95.5 \%$ (42 of 44 isolates) were co-resistant to quinolone antibiotics. In multivariate logistic regression analysis, inability to turn over in bed, diabetes, and invasive procedures within the last 2 years were the only variables independently associated with fecal carriage of CTX-M-type ESBL producers. Nursing home residents in Japan exhibit a high prevalence of CTX-M-type ESBL-producing Enterobacteriaceae carriage, with a high level of co-resistance to quinolones.

Keywords: Enterobacteriaceae, extended-spectrum beta-lactamase (ESBL), risk factors, fecal carriage, nursing homes, Japan

\section{Introduction}

The detection rate of extended-spectrum $\beta$-lactamases (ESBLs) in clinical isolates of Escherichia coli and Klebsiella pneumoniae in the Kinki region of mid-western Japan significantly increased from $0.2 \%$ to $7.3 \%$ and from $0.0 \%$ to $2.4 \%$, respectively, over a 10-year period from 2000 to $2009 .{ }^{1}$ Our recent study among healthy adults in the same region showed $6.4 \%$ prevalence of ESBL producers, most of which were CTX-M-type ESBLs. ${ }^{2}$ The reasons for the increased prevalence of ESBL producers in Japan have not been adequately investigated. In the USA and Europe, nursing homes have been identified as a reservoir of antibiotic-resistant bacteria and possibly play an important role in their emergence and spread. ${ }^{3-5}$ Therefore, we studied the prevalence of, and risk factors associated with, fecal carriage of CTX-M-type ESBL-producing Enterobacteriaceae among the residents of nursing homes. 


\section{Materials and methods}

The study was conducted from March to September 2010 in three nursing homes located in mid-western Japan. Nursing home A was a privately owned 150-bed nursing home, whereas nursing homes $\mathrm{B}$ and $\mathrm{C}$ were 100 - and 50-bed institutions, respectively, run by the local government.

We analyzed 225 stool samples from the three nursing homes, and one sample was obtained from each resident. Data on resident characteristics, including length of stay in the current nursing home, co-morbidities, and past and present antibiotic use, were collected from resident charts. Detailed information was also collected from resident charts in nursing home $\mathrm{A}$, which had the highest prevalence of ESBL-producing Enterobacteriaceae carriers. The information included residents' physical and mental health status, history of invasive procedures within the last 2 years, past and present urinary tract infections, history of stroke, and other health conditions, such as diabetes.

ESBL production was identified by phenotypic and genotypic methods, as described elsewhere. ${ }^{2}$ Collected fecal specimens were plated on MacConkey agar supplemented with $2 \mu \mathrm{g} / \mathrm{mL}$ cefotaxime (CTX-MacConkey) using the streak culture method. ESBL expression was confirmed using cefotaxime and ceftazidime, with and without clavulanic acid, as recommended by the Clinical and Laboratory Standards Institute (CLSI), and each set of samples was tested with CLSI quality control strains E. coli ATCC25922 and K. pneumoniae ATCC700603. ${ }^{6}$ Isolates were identified using conventional biochemical tests. Drug susceptibility tests for imipenem, meropenem, amikacin, gentamicin, ciprofloxacin, norfloxacin, and tetracycline were performed with the disc diffusion method using SN Discs (Nissui Pharmaceutical Co, Ltd, Tokyo, Japan).

Bacterial DNA was extracted from the isolates by boiling the bacterial suspensions. DNA samples with a concentration of $0.1 \mathrm{ng} / \mu \mathrm{L}$ were used as a template for polymerase chain reaction (PCR) analysis. The universal primers CTX-M-U1 (5'-ATG TGC AGY ACC AGT AAR GTK ATG GC-3') and CTX-M-U2 (5'-TGG GTR AAR TAR GTS ACC AGA AYC AGC GG-3') were used to detect the $b l a_{\text {CTX-M }}$ gene, as described previously. ${ }^{7}$ DNA from the reference E. coli $b l a_{\text {СТX-M }}$-positive strain was used as a positive control. To genotype the $b l a_{\text {СТХ-M }}$ genes, we used four primer sets that amplify group-specific $b l a_{\text {CTX-M }}$ genes, as described elsewhere. ${ }^{8}$ To identify the $q n r A, q n r B$, and $a a c\left(6^{\prime}\right)$-Ib genes, PCR analysis was performed on the samples collected from nursing home A, as described elsewhere. ${ }^{9} 10$ PCR products were visualized on $2 \%$ agarose gel electrophoresis followed by staining with GelRed Nucleic Acid Gel Stain (Biotium, Hayward, CA, USA).

Statistical analysis was performed using SPSS v.16.0 (SPSS, Chicago, IL, USA). All qualitative variables were analyzed using the $\chi^{2}$ or Fisher's exact tests, and quantitative variables were analyzed with the Mann-Whitney $U$ test, as appropriate. Factors identified in univariate models as being statistically significant were included in the multivariate logistic regression model and are presented as odds ratios with $95 \%$ confidence intervals. Statistical significance was set at $P<0.05$.

\section{Results}

The characteristics of the 225 study residents from the three nursing homes are listed in Table 1. The median age of the residents was 85 years (range $52-100$ years). The majority $(74.7 \%)$ of the residents were women. The median length of stay in the three nursing homes varied from 87 to 300 days.

Bacterial growth on CTX-MacConkey agar was observed in 103 of 225 samples (45.8\%). The prevalence of CTX-Mtype ESBL-producing Enterobacteriaceae, as confirmed by phenotypic and genotypic methods, was $22.9 \%$ (33 of 144 samples) in nursing home A, $18.8 \%$ (9 of 48 samples) in nursing home $\mathrm{B}$, and $6.1 \%$ (2 of 33 samples) in nursing home C (Table 2). E. coli was the predominant CTX-Mtype ESBL-producing bacterium among these isolates (41 of 44 isolates), followed by other Escherichia spp. (3 of 44 isolates). Genotyping of bla $a_{\text {СTX-M }}$ gene-positive isolates showed that $30(68.2 \%), 13(29.5 \%)$, and $1(2.3 \%)$ of the 44 samples belonged to groups CTX-M-9, CTX-M-1, and CTX-M-2, respectively.

Disc diffusion test for multidrug resistance revealed high levels of co-resistance of CTX-M-type ESBL producers to quinolone antibiotics ( 33 of 33,8 of 9 , and 1 of 2 isolates in nursing homes $\mathrm{A}, \mathrm{B}$, and $\mathrm{C}$, respectively). More than half of these isolates ( 25 of 42) were resistant to tetracycline in addition to quinolones. However, all the 44 CTX-M-type

Table I Characteristics of the nursing home residents

\begin{tabular}{llll}
\hline Variable & \multicolumn{2}{l}{ Nursing home } \\
\cline { 2 - 4 } & A & B & C \\
\hline Capacity & 150 & 100 & 50 \\
No of residents studied & 144 & 48 & 33 \\
Age, years & 85.0 & 86.5 & 84.0 \\
(median [range]) & $(53-100)$ & $(52-100)$ & $(60-98)$ \\
Female sex, \% & 71.5 & 83.3 & 75.8 \\
Length of stay, days & $300 \pm 345$ & $105 \pm 194$ & $87 \pm 136$ \\
(median \pm SD [range]) & $(2-1,490)$ & $(2-1,015)$ & $(8-630)$ \\
\hline
\end{tabular}

Abbreviation: SD, standard deviation. 
Table 2 Prevalence of ESBL-producing Enterobacteriaceae in nursing homes

\begin{tabular}{|c|c|c|c|c|}
\hline \multirow[t]{2}{*}{ Variable } & \multicolumn{3}{|c|}{ Nursing home (n [\%]) } & \multirow{2}{*}{$\begin{array}{l}\text { Total } \\
\text { (n [\%]) }\end{array}$} \\
\hline & A & B & C & \\
\hline No of residents studied & 144 & 48 & 33 & 225 \\
\hline ESBL-producing & $37(25.7)$ & $9(18.8)$ & $3(9.1)$ & $49(21.7)$ \\
\hline \multicolumn{5}{|l|}{ Enterobacteriaceae } \\
\hline CTX-M-type ESBL & $33(22.9)$ & $9(\mid 8.8)$ & $2(6.1)$ & $44(19.6)$ \\
\hline \multicolumn{5}{|l|}{ Bacterial species } \\
\hline Escherichia coli & 31 (93.9) & $8(88.9)$ & $2(100.0)$ & $4 \mathrm{I}(93.2)$ \\
\hline Other & $2(6.0)$ & I (II.I) & $0(0.0)$ & $3(6.8)$ \\
\hline Enterobacteriaceae & & & & \\
\hline
\end{tabular}

Abbreviation: ESBL, extended-spectrum $\beta$-lactamase.

ESBL producers identified were susceptible to carbapenems and amikacin. Only one isolate was susceptible to all the antibiotics tested, and another isolate was resistant to gentamicin and tetracycline only. In nursing home A, where $100.0 \%$ (33 of 33 isolates) quinolone co-resistance was observed, we detected the $a a c\left(6^{\prime}\right)-I b$ gene in 22 of the 33 isolates (66.7\%); however, none of the isolates carried the qnrA or $q n r B$ genes.

Analysis of the data on resident characteristics showed no significant differences in terms of age, gender, or length of stay between carriers and non-carriers of CTX-M-type ESBL producers. Furthermore, carriers and non-carriers of CTXM-producing bacteria did not differ in the current and past antibiotic use (data not shown). However, univariate analysis of the data collected from the resident charts in nursing home A revealed the following risk factors for CTX-M-type ESBL-producing Enterobacteriaceae carriage: (1) resident's condition that requires the highest level of care; (2) inability to turn over in bed; (3) diaper use; (4) diabetes; (5) urinary tract infection; (6) history of hospitalization within the last year; and (7) invasive procedures within the last 2 years (Table 3 ). In multivariate logistic regression analysis, inability to turn over in bed, diabetes, and invasive procedures within the last 2 years were the only variables independently associated with fecal carriage of CTX-M-type ESBL producers.

\section{Discussion}

Our findings show that nursing homes in mid-western Japan have an almost three times higher prevalence $(19.6 \%)$ of CTX-M-type ESBL producers than that reported in the previous clinical study and among healthy people in the same region. ${ }^{1,2}$ Thus, as predicted, nursing homes may play the important role of reservoir in the rapid spread of CTX-M-type ESBLs in Japan. However, the prevalence of fecal carriage of ESBL producers in Japanese nursing homes is low compared
Table 3 Univariate and multivariate logistic regression analyses of risk factors associated with CTX-M-type ESBL-producing Enterobacteriaceae carriage in nursing home $\mathrm{A}$

\begin{tabular}{|c|c|c|c|c|}
\hline \multirow[t]{2}{*}{ Characteristics } & \multicolumn{2}{|c|}{ Univariate analysis } & \multicolumn{2}{|c|}{ Multivariate analysis } \\
\hline & OR $(95 \% \mathrm{Cl})$ & $P$ value & OR $(95 \% \mathrm{CI})$ & $P$ value \\
\hline \multicolumn{5}{|c|}{ Required care level } \\
\hline Level 5 & $\begin{array}{l}3.2 \\
(1.29-7.914)\end{array}$ & 0.009 & - & \\
\hline Level I-4 & I & & & \\
\hline \multicolumn{5}{|c|}{ Ability to turn over in bed } \\
\hline Incapable & $\begin{array}{l}3.50 \\
(1.56-7.86)\end{array}$ & 0.002 & $\begin{array}{l}2.81 \\
(1.18-6.70)\end{array}$ & 0.019 \\
\hline Capable & 1 & & 1 & \\
\hline \multicolumn{5}{|l|}{ Use of diapers } \\
\hline Yes & $\begin{array}{l}2.61 \\
(1.14-5.99)\end{array}$ & 0.021 & - & \\
\hline No & 1 & & & \\
\hline \multicolumn{5}{|l|}{ Diabetes } \\
\hline Yes & $\begin{array}{l}2.78 \\
(1.11-6.99)\end{array}$ & 0.025 & $\begin{array}{l}3.22 \\
(1.11-9.32)\end{array}$ & 0.031 \\
\hline No & 1 & & I & \\
\hline \multicolumn{5}{|c|}{ Past and present urinary tract infection } \\
\hline Yes & $\begin{array}{l}4.13 \\
(1.61-10.56)\end{array}$ & 0.002 & - & \\
\hline No & 1 & & & \\
\hline \multicolumn{5}{|c|}{ History of hospitalization within the last year } \\
\hline Yes & $\begin{array}{l}3.16 \\
(1.37-7.30)\end{array}$ & 0.006 & - & \\
\hline No & 1 & & & \\
\hline \multicolumn{5}{|c|}{ Invasive procedures within the last 2 years } \\
\hline Yes & $\begin{array}{l}4.73 \\
(2.06-10.85)\end{array}$ & $<0.001$ & $\begin{array}{l}4.54 \\
(1.87-\mid 1.01)\end{array}$ & 0.001 \\
\hline No & 1 & & 1 & \\
\hline
\end{tabular}

Notes: Required care level is assessed by the authorized professional people according to the Long-Term Care system of Japan. ${ }^{15}$ Five levels of care are distinguished: care level $I$ is defined as requiring partial care, whereas care level 5 is defined as impossible to live without care.

Abbreviations: $\mathrm{Cl}$, confidence interval; ESBL, extended-spectrum $\beta$-lactamase; OR, odds ratio.

with findings in some other countries. In Northern Ireland, UK, $40.5 \%$ (119 of 294 samples) of nursing home residents were gut carriers of ESBL-producing E. coli, which were also resistant to fluoroquinolones; ${ }^{3}$ in Bolzano, Italy, 41.4\% of 111 residents in a long-term-care facility (LTCF) were colonized with ESBL-producing E.coli. ${ }^{11}$

The dominance of CTX-M among the ESBLs in nursing homes is consistent with the global trend. The prevalent identification of E. coli (93.2\%; 41 of 44 isolates) among the ESBL producers and the CTX-M-9 group dominance $(68.2 \%$; 30 of 44 isolates) is similar to previous findings in Japan, both in clinical settings and among asymptomatic individuals. ${ }^{2,12,13}$

Among the CTX-M-type ESBL-producing Enterobacteriaceae found in nursing homes, 95.5\% (42 of 44 isolates) were co-resistant to quinolone antibiotics. It is 
suggested that $b l a_{\text {СТX-M }}$ genes are directly linked to quinoloneresistance $(q n r)$ genes. However, we did not identify isolates with $q n r A$ or $q n r B$ genes in this study. ${ }^{14} \mathrm{~A}$ hospital study in Japan reported that most of the CTX-M-producing isolates were resistant to fluoroquinolones, ${ }^{13}$ whereas fluoroquinolone resistance was observed in only $7.1 \%$ (1 of 14 isolates) of asymptomatic ESBL carriers in Japan. ${ }^{2}$ However, all the CTX-M-producing Enterobacteriaceae isolated at the nursing homes were susceptible to carbapenems and amikacin.

Poor functional status, diabetes, and invasive procedures have also been previously identified as risk factors for ESBL colonization or infection both among residents of LTCFs and among general patients. ${ }^{4,5}$ Not only residents, but also $11.6 \%$ of 69 staff members in an LTCF were colonized with ESBL-producing E.coli. ${ }^{11}$ This may imply contact transmission of CTX-M-type ESBL-producing Enterobacteriaceae. Furthermore, among the three studied nursing homes, fecal carriage of CTX-M-type ESBL producers was the lowest (6.1\%) in nursing home C (50-bed unit). Infection-control measures may have been better implemented in the nursing home with fewer residents. Therefore, infection control must be closely monitored in nursing homes to prevent residentto-resident, staff-to-resident, and vice versa transmission of ESBL producers.

In summary, nursing home residents in Japan have a high prevalence of CTX-M-type ESBL-producing Enterobacteriaceae carriage, with a high level of co-resistance to quinolones. Further monitoring and public health efforts focusing on nursing homes are needed to control the spread of ESBL-producing bacteria in Japan's aging society.

\section{Acknowledgments}

This work was supported in part by KAKENHI (Grant-in-Aid for Exploratory Research), the Ministry of Education, Culture, Sports, Science and Technology, Japan, and the Japan Science and Technology Agency (JST)/Japan International Cooperation Agency (JICA) as part of the Science and Technology Research Partnership for Sustainable Development (SATREPS). The excellent technical assistance of Misako Kikuchi is acknowledged.

\section{Disclosure}

The authors report no conflicts of interest in this work.

\section{References}

1. Nakamura T, Komatsu M, Yamasaki K, et al. Epidemiology of Escherichia coli, Klebsiella species, and Proteus mirabilis strains producing extended-spectrum beta-lactamases from clinical samples in the Kinki Region of Japan. Am J Clin Pathol. 2012;137(4):620-626.

2. Luvsansharav UO, Hirai I, Niki M, et al. Prevalence of fecal carriage of extended-spectrum beta-lactamase-producing Enterobacteriaceae among healthy adult people in Japan. J Infect Chemother. 2011;17(5): 722-725.

3. Rooney PJ, O'Leary MC, Loughrey AC, et al. Nursing homes as a reservoir of extended-spectrum beta-lactamase (ESBL)-producing ciprofloxacin-resistant Escherichia coli. J Antimicrob Chemother. 2009;64(3):635-641.

4. Bonomo RA. Multiple antibiotic-resistant bacteria in long-term-care facilities: an emerging problem in the practice of infectious diseases. Clin Infect Dis. 2000;31(6):1414-1422.

5. Oteo J, Pérez-Vézquez M, Campos J. Extended-spectrum [beta]lactamase producing Escherichia coli: changing epidemiology and clinical impact. Curr Opin Infect Dis. 2010;23(4):320-326.

6. National Committee for Clinical Laboratory Standards. Performance standards for antimicrobial susceptibility testing. Document M100S14 ed. 14 (2004). Wayne (PA): NCCLS; 2004.

7. Monstein HJ, Ostholm-Balkhed A, Nilsson MV, Nilsson M, Dornbusch K, Nilsson LE. Multiplex PCR amplification assay for the detection of blaSHV, blaTEM and blaCTX-M genes in Enterobacteriaceae. APMIS. 2007;115(12):1400-1408.

8. Pitout JD, Hossain A, Hanson ND. Phenotypic and molecular detection of CTX-M-beta-lactamases produced by Escherichia coli and Klebsiella spp. J Clin Microbiol. 2004;42(12):5715-5721.

9. Robicsek A, Strahilevitz J, Sahm DF, Jacoby GA, Hooper DC. qnr prevalence in ceftazidime-resistant Enterobacteriaceae isolates from the United States. Antimicrob Agents Chemother. 2006;50(8):2872-2874.

10. Park CH, Robicsek A, Jacoby GA, Sahm D, Hooper DC. Prevalence in the United States of $a a c\left(6^{\prime}\right)-I b-c r$ encoding a ciprofloxacin-modifying enzyme. Antimicrob Agents Chemother. 2006;50(11):3953-3955.

11. March A, Aschbacher R, Dhanji H, et al. Colonization of residents and staff of a long-term-care facility and adjacent acute-care hospital geriatric unit by multiresistant bacteria. Clin Microbiol Infect. 2010;16(7): 934-944.

12. Shibata N, Kurokawa H, Doi Y, et al. PCR classification of CTX-M-type beta-lactamase genes identified in clinically isolated gram-negative bacilli in Japan. Antimicrob Agents Chemother. 2006;50(2):791-795.

13. Chong Y, Yakushiji H, Ito Y, Kamimura T. Clinical and molecular epidemiology of extended-spectrum beta-lactamase-producing Escherichia coli and Klebsiella pneumoniae in a long-term study from Japan. Eur J Clin Microbiol Infect Dis. 2011;30(1):83-87.

14. Chong Y, Ito Y, Kamimura T. Genetic evolution and clinical impact in extended-spectrum beta-lactamase-producing Escherichia coli and Klebsiella pneumoniae. Infect Genet Evol. 2011;11(7):1499-1504.

15. Mitchell OS, Piggott J, Shimizutani S. Aged-care support in Japan: perspectives and challenges. Benefits Q. 2006;22(1):7-18.
Infection and Drug Resistance

\section{Publish your work in this journal}

Infection and Drug Resistance is an international, peer-reviewed openaccess journal that focuses on the optimal treatment of infection (bacterial, fungal and viral) and the development and institution of preventive strategies to minimize the development and spread of resistance. The journal is specifically concerned with the epidemiology of antibiotic

\section{Dovepress}

resistance and the mechanisms of resistance development and diffusion in both hospitals and the community. The manuscript management system is completely online and includes a very quick and fair peerreview system, which is all easy to use. Visit http://www.dovepress.com/ testimonials.php to read real quotes from published authors. 